\title{
Performance on a Food Health Assessment Using Emoticons with Pre-Literacy-Aged Children
}

\author{
Gregory J. Privitera*, Stephanie I. Vogel, Danielle E. Antonelli \\ Department of Psychology, Saint Bonaventure University, New York, USA \\ *Corresponding author: gprivite@sbu.edu
}

Received April 13, 2013; Revised April 22, 2013; Accepted April 27, 2013

\begin{abstract}
In the present study, we tested the hypothesis that 3-year-old children at a pre-literacy age can correctly identify healthy and unhealthy foods if they are paired with emoticons to convey this information. Using a multiple baseline single case design, 6 boys were shown pairs of cards depicting one healthy and one unhealthy food, across many trials. Children were asked to point to the healthy food on half the trials and to point to the food that was not healthy on the other half of trials. On half the trials, the foods depicted were familiar to the child; on the other half of trials, the foods were not familiar. A happy face emoticon was displayed on all cards depicting a healthy food; a sad face emoticon was displayed on all cards depicting an unhealthy food. If a child did not score $100 \%$ on each trial in baseline, then he was given a lesson to teach him how to relate emoticons with health and tested again in a postbaseline phase. The results showed that four of six children scored $100 \%$ on this assessment in baseline. The two remaining children scored $100 \%$ in the post-baseline phase. These results show that pre-literacy-aged children can readily match concepts of health (healthy, not healthy) to emotional correlates of health (healthy-good, not healthy-not good). At present, these are the first results to show that literacy is not an absolute requirement for children to identify the healthfulness of foods, and could lead to possible early interventions that could be easily incorporated into a preschool curriculum for children as young as 3-years of age.
\end{abstract}

Keywords: child health, health education, literacy, preschool, food

\section{Introduction}

According to the Centers of Disease Control, rates of obesity have more than doubled since 1980 from $5 \%$ to $10.4 \%$ among preschool-aged children two to five years of age [1]. One possible contributing factor to this rise is that preschool children cannot read and write, and so are unable to identify health information. The ability to understand health information, to include nutrition-food health information, is called health literacy [2,3]. The American Medical Association (AMA), Institute of Medicine (IOM), and the U.S. Department of Health and Human Services (HHS) similarly define health literacy as a set of skills (e.g., basic reading and math/counting skills) needed to acquire knowledge about health and make appropriate health-related decisions $[4,5,6]$.

This current model of health literacy implies that it is not possible to teach concepts of health to 3-year-old children because they cannot yet read or write. However, it would be advantageous to establish a method by which concepts of health could be conveyed to potentially address the growing obesity rates among this age group. One possible method by which concepts of health could be conveyed to children is by relating these concepts to a skill that is expressed among children at this ageemotion. The ability for children to use and recognize emotional cues is regarded as a key ability in their social development at three years of age [7]. Two to five year old children can accurately respond to and identify expressions of their emotions and those of their peers $[8,9]$, can appropriately recognize emoticons on pictorial scales $[10,11]$, and can effectively rate their liking for foodstuffs using these pictorial scales [12]. Hence, preschool children express cognitive (the ability to understand emotion) and behavioral (the ability to make appropriate emotion-related decisions) "literacy" or knowledge of emotion.

We surmised, then, that we could communicate concepts of health as being "good for you" or "not good for you" to preschool children by relating these concepts of health to emotional correlates of health, i.e., healthygood, not healthy-not good. To relate the healthfulness of food with emotional correlates of health, emoticons (happy, sad) were displayed on picture cards with foods (healthy, not healthy) that were either familiar or unfamiliar to 3-year-old children in a preschool. These cards were shown to the children who made judgments regarding the healthfulness of those foods. We expected that 3 -year-old children could correctly identify pictures of healthy and unhealthy foods if these foods were paired with emoticons to convey this information.

\section{Methodology}

\subsection{Participants}

A collective sample of six boys in a preschool program at a school in the Western New York region was selected. 
Due to the single case nature of the study, only boys were observed so that single case comparisons could be made with control for gender differences. The single case design was used because the class size at the school was too small to create a grouped design and because individualized picture cards had to be created for each child and so an analysis at the level of the individual was warranted. The age, weight, height, and BMI of each child are given in Table 1. BMI percentiles were computed using Centers for Disease Control and Prevention (CDC) normative data [13]. At least one parent of each child was a college graduate. Each family lived within 15 miles of the school and earned a median annual income of about $\$ 50,000$ U.S. dollars, similar to median income in the U.S. Parents also completed a parent-child health questionnaire to indicate their level of agreement to eight statements about the extent to which they communicate about health with their children. Each scale ranged from 1 (never) to 4 (very often), with higher scores indicating more frequent communication between the parent and child about health. Total scores on this measure, which ranged from 8 to 32 , are also given in Table 1 .

Table 1. Child demographic data

\begin{tabular}{ccccccc}
\hline Child & $\begin{array}{c}\text { Child } \\
\text { Height } \\
\text { (inches) }\end{array}$ & $\begin{array}{c}\text { Child } \\
\text { Weight } \\
\text { (pounds) }\end{array}$ & $\begin{array}{c}\text { Child Age } \\
\text { (years, } \\
\text { months) }\end{array}$ & $\begin{array}{c}\text { Child } \\
\text { BMI }\end{array}$ & $\begin{array}{c}\text { BMI } \\
\text { Percentile }\end{array}$ & $\begin{array}{c}\text { Parent- } \\
\text { Child } \\
\text { Health } \\
\text { Score }\end{array}$ \\
\hline A & 37 & 33 & 3,10 & 16.9 & 84 & 21 \\
B & 38 & 34 & 3,11 & 16.6 & 76 & 23 \\
C & 37.5 & 29 & 3,7 & 14.5 & 10 & 31 \\
D & 39 & 36 & 3,9 & 16.6 & 77 & 29 \\
E & 36 & 32 & 3,8 & 17.4 & $89^{*}$ & 24 \\
F & 40 & 39 & 3,8 & 17.1 & $86^{*}$ & 24
\end{tabular}

A healthy BMI range for 3-year old children is the 5th to 85th percentile. In the second to last column: * indicates a BMI in the overweight range of the BMI. BMI percentiles were computed with adjustments made for age and sex based on the Centers for Disease Control and Prevention (CDC) normative data (Kuczmarski et al., 2002)

\subsection{Procedures}

A multiple baseline design was used with four phases: preliminary, baseline, intervention, and post-baseline phase. A multiple baseline single case design was used because the intervention involved learning and so performance was not expected to return to baseline. The university's Institutional Review Board approved all procedures for this study.

Preliminary phase. The parents of each child consented for their child to participate in this study. Upon giving consent, parents completed a demographic survey and indicated which foods their child had consumed in his lifetime using a list of over 200 foods to choose from. Based on the parent responses to this survey, 48 food cards were created for each individual child, with half the cards depicting familiar foods (foods the child had eaten before) and half the cards depicting unfamiliar foods (foods the child had not eaten before). Hence, familiar and unfamiliar in this study refers to familiarity with eating the foods. The children were shown pairs of cards with one picture of a food and one emoticon on each card; a sample pair of food cards is shown in Figure 1.
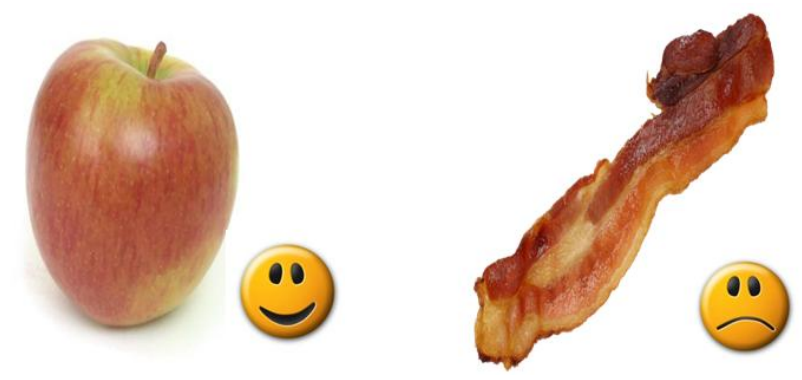

Figure 1. A sample pair of food cards used in the study. One card was a healthy food with a happy face in the bottom right corner (left); the second card was an unhealthy food with a sad face in the same location (right). Pairs of food cards were individually created for each child based upon parent responses in the preliminary survey

Baseline phase. The baseline phase was conducted in two or four trials with one child observed at a time. In each trial, a child was shown 12 pairs of food cards one pair at a time. Half the pairs (six pairs) were foods their parents identified as familiar; the other half of pairs (six pairs) were foods their parents identified as unfamiliar. In each pair, one card was a healthy food with a happy face emoticon in the bottom right corner; the other card was an unhealthy food with a sad face emoticon in the bottom right corner. Studies show that children can relate or match "happy" and "sad" emoticons with their liking of foods and drinks [12]. Therefore, to avoid possible confusion we matched the emoticons used with the connotative meaning of healthy foods being "good for you" (happy, good) and unhealthy foods being "not good for you" (sad, not good). A total of two trials were conducted each day, so baseline could last one or two days depending on the number of trials conducted.

For half the pairs, a child was asked, "Please point to the food that is healthy." For the remaining pairs, the child was asked, "Please point to the food that is not healthy." The phrase not healthy was used in place of unhealthy because many 3-year-old children have not yet been taught prefixes and show difficulty understanding the meaning of prefixes [14]. Also, preschool-aged children can relate the meaning of words based on the prosody (i.e., the tone of voice, rhythm, and pitch) of the questions asked [15]. To avoid a possible confound of prosody, the researcher asked all questions in a monotone voice to standardize prosody in each trial. Once a child pointed to a food card, their choice was recorded. The dependent measure was the total percentage of correct responses per trial using familiar foods and using unfamiliar foods (i.e., the number of correct responses divided by six pairs of cards shown in each familiar-unfamiliar trial).

According to parent surveys given in the preliminary phase, each child in this study had some familiarity with the concept of "health." We therefore anticipated that some children might, without an intervention, immediately relate the emoticons with the positivenegative connotations that are often communicated when describing health. Children who scored $100 \%$ on the health assessment in baseline were given four baseline trials and were not tested further. Child C, D, E, and F listed in Table 1 met this criterion. To assess health knowledge further with Child A and B, who scored near chance (i.e., about $50 \%$ ) on each trial in baseline, the number of trials in baseline was staggered as required using a multiple baseline design; i.e., two trials for Child 
A (one baseline day) and four trials for Child B (two baseline days). Staggered baseline trials are used to minimize the likelihood that the simple passage of time can explain changes, if any, following the intervention phase.

Intervention phase. The day following the last baseline day was the intervention. Hence, Child A received the lesson on Day 2; Child B received the lesson on Day 3. The intervention was a "faces of health" lesson to teach children how to relate emoticons with healthy and unhealthy foods. The researcher played the role of teacher. The lesson used a teacher-directed lesson plan to ensure that each child experienced similar interactions and attention from the teacher who gave the lesson. The lesson was 10 minutes long. First, each child was taught that healthy food is "good for your body" and that a food that is not healthy is "not as good for your body." Each child was never told that unhealthy food is "bad for your body" because the word "bad" can have moral (as opposed to strictly factual) undertones that children as young as 3years-old can recognize and identify [16]. Next, each child was shown a happy face emoticon and asked if being happy makes them feel good or not good. Both children replied that it makes them feel good. Each child was then shown a sad face emoticon and asked if being sad makes them feel good or not good. Both children replied that it makes them feel not good. Finally, each child was told that since being happy and eating healthy foods are good, then we can use a happy face to represent healthy foods; since being sad and eating foods that are not healthy is not good, then we can use a sad face to represent foods that are not healthy. The children were then shown one novel healthy-unhealthy food card pair with emoticons on the cards and asked to correctly identify the healthy and unhealthy food. The lesson ended when both children correctly identified the healthy and unhealthy food picture card on the first trial in both cases.

Post-baseline Phase. Approximately one-hour after the intervention was given, Child A and B were retested with the same baseline assessment except that a new set of 12 pairs of food cards were used in which, half the foods were familiar and half were unfamiliar to each child according to parent reports in the preliminary survey. The new cards were used to ensure that any change in response accuracy from baseline to post-baseline was specifically due to the intervention and not to possible testing effects. Post-baseline ended when a total of six trials were completed (including baseline). The total percentage of correct responses per trial in this phase was compared to those observed in baseline.

\section{Results}

Child C, D, E, and F scored $100 \%$ on all trials in baseline. It was assumed that these four children would continue to score $100 \%$ on all subsequent trials and so were not tested further. These four children were asked after the last baseline trial how they knew the answers. Interestingly, all four children replied that the foods were happy or sad, indicating that the children used the emoticons to gauge their answers.

Child A and B scored at or near 50\% correct on each trial in baseline; their baseline data are shown in Figure 2.
To identify if the "faces of health" intervention was effective, we used the criteria of observing a change in level between baseline and post-baseline. As shown in Figure 2, both children who scored at or near $50 \%$ in baseline scored $100 \%$ on all post-baseline trials following the intervention. This pattern is clear evidence of a change in level because the change occurred only after the intervention following a baseline of either two or four trials. Child A and B were asked after the last baseline trial and again after the last post-baseline trial how they knew the answers. Interestingly, both children could not explain how they were responding after baseline, but both children replied that the foods were happy or sad after the last post-baseline trial. Hence, these two children used the emoticons to gauge their answers in the post-baseline phase, but not in the baseline phase.

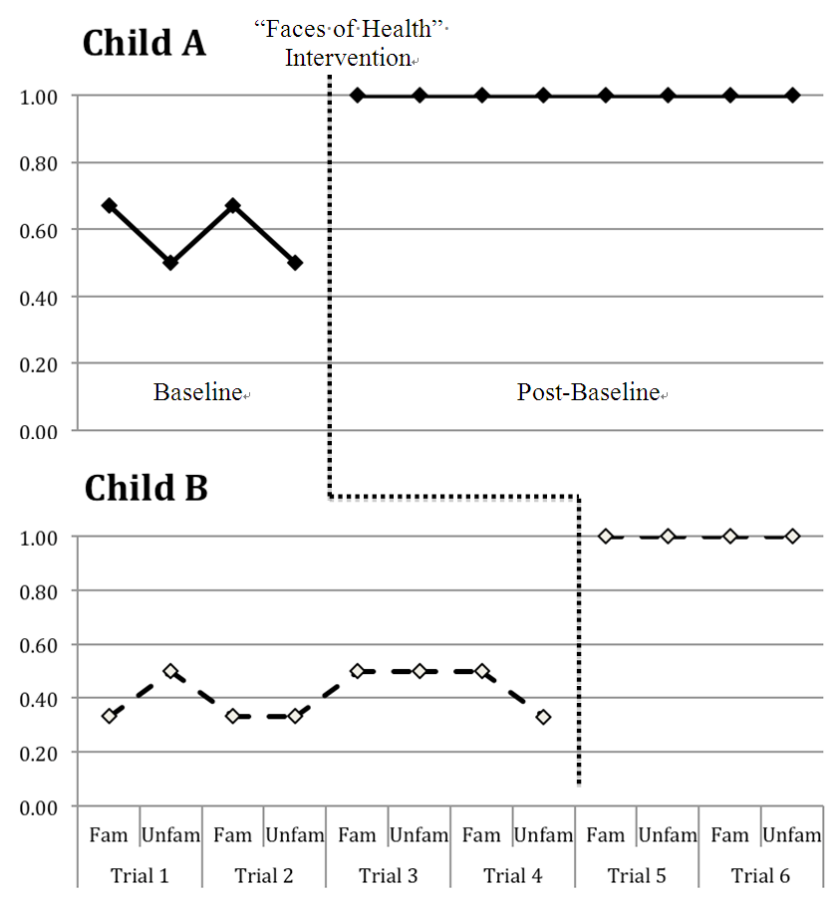

Figure 2. Multiple baseline data for two 3 year-old children in baseline (to the left of the dashed horizontal line) and post-baseline (to the right of the dashed horizontal line). The dashed horizontal line represents the intervention. A change in level is evident only at the time baseline ends and after the intervention. Both children scored at chance (about 50\%) on all trials before the "faces of health" intervention; both children scored $100 \%$ on all trials following the intervention. Two trials were conducted each day

\section{Discussion}

The hypothesis that pre-literate 3-year-old children can identify foods as being healthy or not healthy if these foods are paired with emoticons to convey this information was tested. The results show that children used emoticons to correctly identify foods as being healthy and unhealthy. Interestingly, four of the six children immediately related happy expressions of emotion to healthy foods and related sad expressions of emotion to unhealthy foods. These data suggest that many children who are at a pre-literacy age may readily use emotional correlates of health as being good for you (healthy foods) and not being good for you (not healthy foods). Even among children who did not score $100 \%$ in 
baseline, it only took one 10-minute lesson in an intervention phase to get accuracy to $100 \%$ in postbaseline. By any measure of learning, these findings suggest that pre-literacy-aged children can readily relate healthy and unhealthy foods to corresponding happy-sad emotions. Such findings extend results in prior studies showing that children can readily use emotion to develop social skills and social understanding [16,17,18], by showing that children can also relate emotion to categories of foods as being "healthy" and "not healthy."

Due to the nature of the design used, some limitations or cautions can be identified. First, the results do not generalize to a population and may be specific to boys because girls were not observed in this study. However, the multiple baseline design does allow for generalization across participants. In this study, all six children (four without and two with intervention) scored $100 \%$ on a health assessment, even when foods were unfamiliar to them. Second, all pictures cards had a picture of a food and a corresponding emoticon, and all children-either in baseline (Child C, D, E, and F) or in post-baseline (Child A and B)-identified the foods as being "happy or sad." Hence, the children were clearly using the emoticons to gauge their responses, and did not necessarily demonstrate conceptual knowledge of health. Regardless, most health literacy models infer that communicating health knowledge to this age group will not be possible because they lack basic reading and writing skills. The results presented here, however, demonstrate that some information about health, i.e., healthy-good, not healthynot good can be conveyed so long as each food has a corresponding emoticon to help the child gauge their judgment.

Interestingly, the two children who scored near 50\% in baseline were both in the healthy range of the BMI. Of those who scored $100 \%$ in baseline, two were in the healthy range and two were in the overweight range of the BMI. Hence, learning the emoticon-food category pairing did not appear to vary by BMI. Instead, one pattern that did emerge was that parents of the two children who scored near $50 \%$ in baseline communicated least frequently with their child about health, summarized in Table 1 (last column). Hence, it may be that the ability to relate health information to expressions of emotion is related to the extent to which health is communicated to children by their parents. The intervention in the present study identifies a formal way in which health can be communicated, even by parents, to help children correctly identify categories of foods as being "healthy" and "not healthy."

The findings reported here are significant inasmuch as children at age three are not capable of health literacy according to the AMA, IOM, and HHS [4,5,6]. Using emoticons, then, to communicate basic concepts of health at this age could have important benefits to help children identify foods as being good or not good for you. If children can use emotional correlates of health to correctly identify if a food is healthy or not healthy, then using the "faces of health" method could effectively be used to teach children about categories of health prior to kindergarten. Whether such findings extend to a sample of girls should be tested, as possible gender differences in emotional processing have been reported $[19,20]$.
The extent to which children can use emoticons to correctly identify specific nutrients in foods (such as fat and sugar content) or possibly use emotional correlates of health to shift their choices to healthier foods can also be tested. If pairing healthy foods with happy emoticons can increase a child's choice for healthier foods, then the strategy demonstrated here could potentially be used to supplement nutrition labels for foods that are marketed or aimed at children. At present, however, these data demonstrate that pre-literate children can correctly identify healthy and unhealthy foods if pictures of these foods, whether familiar or not, are matched with the emotional correlates of health (e.g., healthy, happy-good; not healthy, sad-not good).

\section{Conclusion}

The results presented here show preliminary evidence that health information can be communicated to preschool children who cannot yet read or write. Health information was communicated inasmuch as the children could correctly recognize healthy and unhealthy food images when aided with emoticons to guide their judgments. If preschool children can use emotional correlates of health to correctly identify the healthfulness (healthy, not healthy) of a food, then such a method could be used to teach children about health prior to kindergarten. Furthermore, such a method could empower children with the ability to make food choices based on the healthfulness of the foods they choose - such a possibility is now being tested. At present, these are the first results to show that literacy is not an absolute requirement for children to identify the healthfulness of foods. These results are important given the rising rates of obesity, even among preschool-aged children, and could lead to possible early interventions to promote healthy eating among children; interventions that could be easily incorporated into a preschool curriculum for children as young as 3-years of age.

\section{Acknowledgements}

This research was funded, in part, by an internal faculty research grant awarded to the first author.

\section{Statement of Competing Interests}

The authors declare that they have no competing interests.

\section{References}

[1] Ogden, C., \& Carroll, M. Centers for Disease Control. Prevalence of obesity among children and adolescents: United States, trends 1963-1965 through 2007-2008. Retrieved on June 4, 2012 from http://www.cdc.gov/nchs/data/hestat/obesity_child_07_08/obesity _child_07_08.htm.

[2] Drummond, M., \& Drummond, C. (2010). Interviews with boys on physical activity, nutrition, and health: Implications for health literacy. Health Sociological Review, 19, 491-504.

[3] Baker D. W. (2006). The meaning and the measure of health literacy. Journal of General Internal Medicine, 21, 878-883. 
[4] Ad Hoc Committee on Health Literacy (1999). Health literacy: Report of the council on scientific affairs, Journal of the American Medical Association, 281, 552-557.

[5] U.S. Department of Health and Human Services. (2000). Healthy People 2010: Understanding and improving health. Chapter 11. $2^{\text {nd }}$ Ed. Washington, DC: U.S. Government Printing Office.

[6] nstitute of Medicine. (2004). Health literacy: A prescription to end confusion. Washington, DC: National Academies Press.

[7] Denham, S. A., Blair, K., DeMulder, E., Levitas, J., Sawyer, K., Auerbach-Major, S., et.al. (2003). Preschool emotional competence: Pathway to social competence? Child Development, 74, 238-256.

[8] Mathieson, K., \& Banerjee, R. (2010). Preschool peer play: The beginnings of social competence. Educational \& Child Psychology, 27, 9-20.

[9] Slomkowski, C., \& Dunn, J. (1996). Young children's understanding of other people's beliefs and feelings and their connected communication with friends. Developmental Psychology, 32, 442-447.

[10] Bradley, M. M., \& Lang, P. J. (1994). Measuring emotion: The Self-Assessment Manikin and the semantic differential. Journal of Behavior Therapy \& Experimental Psychiatry, 25, 49-59.

[11] Visser, N., Alant, E., \& Harty, M. (2008). Which graphic symbols do 4-year-old children choose to represent each of the four basic emotions. Augmentative and Alternative Communication, 24, 302312.

[12] Capaldi, E. D., \& Privitera, G. J. (2008). Decreasing dislike for sour and bitter in children and adults. Appetite, 50, 139-145.
[13] Kuczmarski, R. J., Ogden, C., Guo, S. S., Grummer-Strawn, L. M., Flegal, K. M., Mei, Z., et al. (2002). CDC growth charts for the United States: Methods and development. Vital and Health Statistics, 11(246), 1-190.

[14] Kuczaj, S. A. (1979). Evidence for language learning strategy: On the relative ease of acquisition of prefixes and suffixes. Child Development, 50, 1-13.

[15] Herold, D. S., Nygaard, L. C., Chicos, K. A., \& Namy, L. L. (2011). The developing role of prosody in novel word interpretation. Journal of Experimental Child Psychology, 108, 229-241.

[16] Smetana, J. G. (2006). Social domain theory: Consistencies and variations in children's social and moral judgments (pp. 69-91). In Killen, M., \& Smetana J. G. (eds.), Handbook of Moral Development. Mahwah, NJ: Erlbaum.

[17] Denham, S. A., Zoller, D. \& Couchoud, E. A. (1994). Socialization of preschoolers' emotion understanding. Developmental Psychology, 30(6), 928-936.

[18] Dunn, J. (2000). Mind-reading, emotion understanding, and relationships. International Journal of Behavioral Development. 24(2), 142-144.

[19] Bosacki, S. L. (2007). Children's understandings of emotion and self: Are there gender differences? Journal of Research in Childhood Education, 22(2), 155-172.

[20] McClure, E. B. (2000). A meta-analytic review of sex differences in facial expression processing and their development in infants, children, and adolescents. Psychological Bulletin, 126(3), 424-453. 